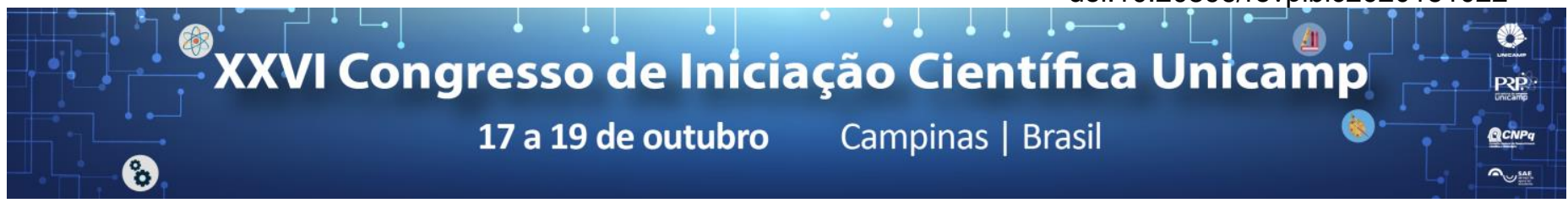

\title{
Análise qualitativa de soluções de sistemas de equações diferenciais ordinárias e aplicações.
}

\section{Mateus Santos Rocha*, Bianca Morelli Rodolfo Calsavara}

\section{Resumo}

Para muitos sistemas de equações diferenciais ordinárias não é possível ou é muito difícil obter explicitamente sua solução. Porém, é possível obter informações sobre estas soluções realizando um estudo qualitativo do sistema sem resolvê-lo. Neste trabalho é feito um estudo de ferramentas que permitam tais análises, como uso de plano de fase e de funções de Liapunov. Além disso, serão estudadas algumas aplicações destas ferramentas.

\section{Palavras-chave:}

Equações diferenciais ordinárias, Análise qualitativa de equações diferenciais, Aplicações de equações diferenciais.

\section{Introdução}

Problemas de diversas áreas do conhecimento, como física, engenharias e biologia, podem ser descritos por sistemas de equações diferenciais. Apesar de o Teorema da existência e unicidade para equações diferenciais ordinárias nos garantir que, sob certas condições, existe única solução para problemas de valor inicial, muitas vezes é muito difícil (ou até impossível) encontrá-la explicitamente.

Por este motivo, neste projeto foram estudados métodos para análise qualitativa de soluções de sistemas de equações diferenciais ordinárias. Foram estudados, por exemplo, planos de fase para sistemas $2 \times 2$, Teorema de Poincaré-Bendixon, Alternativa de Fredholm, funções de Liapunov, bem como aplicações destas ferramentas em sistemas que descrevem a dinâmica presa-predador, o movimento de um pêndulo e um sistema RLC, entre outros.

\section{Resultados e Discussão}

Para um sistema de equações diferenciais lineares com coeficientes constantes da forma $\dot{x}=A x+b$, onde $A$ é uma matriz quadrada $n x n$ e $b$ é uma matriz coluna $n \times 1$, garantimos que existe uma única solução.

Em particular, foi visto que este sistema possui uma solução periódica com período $T$ se, e somente se,

$$
\int_{0}^{T} A^{t} y(t) b d t=0
$$

onde $y(t)$ é solução do sistema adjunto $\dot{y}=-y A(t)$.

Em seguida, foram estudados sistemas autônomos no plano, que são sistemas da forma

$$
\left\{\begin{array}{l}
\dot{x}=f(x, y) \\
\dot{y}=g(x, y)
\end{array}\right.
$$

Uma singularidade para este sistema é um par $\left(x_{0}, y_{0}\right) \in \mathbb{R}^{2}$ tal que $f\left(x_{0}, y_{0}\right)=g\left(x_{0}, y_{0}\right)=0$. As soluções $(x(t), y(t))$ são curvas parametrizadas no plano de fases $(x, y)$ denominadas órbitas. O sistema acima possui significado geométrico entendendo $(f(x, y), g(x, y))$ como um campo vetorial no plano e as órbitas como curvas integrais deste campo, ou seja, as curvas que em cada ponto são tangentes ao campo do sistema

O conjunto

$$
\gamma=\gamma(P)=\{(x(t), y(t)): t \in \mathbb{R}\},
$$

onde $(x(t), y(t))$ é solução do sistema acima sujeito à condição inicial $P=\left(x\left(t_{0}\right), y\left(t_{0}\right)\right)$ é chamado de órbita do sistema que passa por $P$. Define-se então o conjunto $\omega$ limite da órbita que passa por $\mathrm{P}$ como

$\omega(P)=\left\{(x, y) \in \mathbb{R}^{2}: \exists t_{n} \rightarrow+\infty t . q .(x, y)=\lim _{n \rightarrow \infty}\left(x\left(t_{n}\right), y\left(t_{n}\right)\right)\right\}$ O Teorema de Poincaré-Bendixson nos permite obter informações sobre o conjunto $\omega$-limite a partir das órbitas e das singularidades de um sistema. Por exemplo, se $\mathrm{Y}(\mathrm{P})$ é limitada e $\omega(P)$ não contém nenhuma singularidade, então $\omega(P)$ é uma órbita fechada, podendo concluir-se que as soluções que passam por $P$ tendem a ser "cíclicas" à medida que t cresce.

Finalmente, para um sistema autônomo $\dot{x}=f(x)$ de $n$ equações com a condição inicial $f(0)=0$, uma função $V: \Omega \subset \mathbb{R}^{n} \rightarrow \mathbb{R}$ definida em um conjunto $\Omega$ que contém uma bola fechada centrada na origem, é dita de Liapunov para este sistema se $V(0)=0, V(x)>0 \forall x \neq 0$ e $\dot{V}(x) \leq 0 \forall x$, onde $\dot{V}: \Omega \rightarrow \mathbb{R}$ é definida por

$$
\dot{V}(x)=\langle(\operatorname{grad} V)(x), f(x)\rangle
$$

A existência de uma função de Liapunov garante a estabilidade da solução nula, além de restringir o conjunto $\omega$-limite ao conjunto onde esta função de Liapunov possui pontos criticos.

\section{Conclusões}

Dado um sistema autônomo de equações diferenciais podemos linearizá-lo e usar o teorema de PoincaréBendixson para estudarmos o comportamento de suas soluções. Também podemos utilizar de Funções de Liapunov para determinar a estabilidade da solução nula. Além de ser possível determinar a existência de solução periódica de um sistema utilizando o sistema adjunto. Assim, podemos obter diversas informações sobre as soluções de um sistema autônomo mesmo sem encontrá-las explicitamente.

FIGUEIREDO, Djairo Guedes de. Equações Diferenciais Aplicadas.(3a. Edição). Rio de Janeiro: IMPA, 2014.

SOTOMAYOR, Jorge. Equações diferenciais ordinárias. São Paulo: Editora Livraria da Física, 2011.

SOTOMAYOR TELO, Jorge Manuel, Lições de equações diferenciais ordinárias. Rio de Janeiro: IMPA, 1979. 